\title{
ESTRESSORES DA PÓS-GRADUAÇÃO: REVISÃO INTEGRATIVA DA LITERATURA*
}

\author{
Flaviane Cristina Rocha Cesar ${ }^{1}$, Elyana Teixeira Sousa ${ }^{2}$, Luana Cássia Miranda Ribeiro ${ }^{3}$, Lizete Malagoni de \\ Almeida Cavalcante Oliveira ${ }^{3}$
}

\begin{abstract}
RESUMO: Objetivo: identificar evidências científicas relacionadas aos estressores enfrentados por estudantes de mestrado e doutorado durante a sua formação. Metodologia: revisão integrativa da literatura, realizada em outubro de 2017, na Biblioteca Virtual em Saúde, Web of Science, Psychology Information e PubMed. Foram incluídos artigos primários, publicados em inglês, português e espanhol e que contemplassem o tema. Resultados: os resultados compuseram 12 estudos, dos quais emergiram três categorias de estressores: individuais, acadêmicos e de relações interpessoais. Dessas categorias, os estressores que se destacaram foram alta carga de atividades acadêmicas, renda, conflito pós-graduação-família-trabalho e relação aluno-orientador. Conclusão: o desafio para o pós-graduando é conciliar o estresse vivido nesse momento da vida, as atividades acadêmicas e não acadêmicas. Os programas de pós-graduação necessitam desenvolver produção científica de qualidade, que não resulte no adoecimento dos envolvidos nesse processo.

DESCRITORES: Estudantes; Educação de pós-graduação; Estresse psicológico; Saúde mental; Revisão.
\end{abstract}

\section{GRADUATE SCHOOL STRESSORS: AN INTEGRATIVE LITERATURE REVIEW}

\begin{abstract}
Objective:To identify the scientific evidence related to the stressors faced by Master's and PhD students during their graduate program. Methodology:This was an integrative literature review conducted in October 2017 in the Virtual Library of Healthy, Web of Science, Psychology Information and PubMed databases.The review included primary articles on the topic published in English, Portuguese, and Spanish. Results:Twelve studies were analyzed, yielding three categories of stressors: personal, academic, and interpersonal relationships. Of these categories, emphasis goes to high academic workload, income, reconciling graduate studies with family and work, and the student-advisor relationship. Conclusion: The challenge for graduate students lies in reconciling the stress experienced at this time of life, academic activities, and nonacademic activities. Graduate programs need to develop highquality scientific production that does not result in the loss of health of those involved in the process.
\end{abstract}

DESCRIPTORS: Students; Education, Graduate; Stress, Psychological; Health, Mental; Review.

\section{ESTRESORES EN EL POSGRADO: REVISIÓN INTEGRATIVA DE LA LITERATURA}

RESUMEN: Objetivo: Identificar evidencias científicas relacionadas a los estresores enfrentados por los estudiantes de maestría y doctorado durante su formación. Metodología: Revisión integrativa de literatura, realizada en octubre de 2017, en la Biblioteca Virtual em Saúde, Web of Science, Psychology Information y PubMed. Fueron incluidos artículos primarios, publicados en inglés, portugués y español, enfocados en el tema. Resultados: Los resultados entregaron 12 estudios, de los cuales emergieron tres categorías de estresores: individuales, académicos y de relaciones interpersonales. De dichas categorías, los estresores que tomaron mayor relevancia fueron la alta carga de actividades académicas, ingresos, conflicto posgrado-familia-trabajo y relación alumno-tutor. Conclusión: El desafío para el estudiante de posgrado es conciliar el estrés vivido en ese momento de su vida, las actividades académicas y no académicas. Los programas de posgrado necesitan desarrollar producción científica de calidad que no se vea afectada por padecimientos de los involucrados en ese proceso.

DESCRIPTORES: Estudiantes; Educación de Posgrado; Estrés Psicológico; Salud Mental; Revisión.

\footnotetext{
*Artigo extraído da dissertação de mestrado "Qualidade de vida de estudantes de pós graduação stricto sensu da área da saúde". Universidade Federal de Goiás, 2018.
}

${ }^{1}$ Enfermeira. Mestranda em Enfermagem. Universidade Federal de Goiás. Goiânia, GO, Brasil.

${ }^{2}$ Enfermeira. Doutoranda em Enfermagem. Universidade Federal de Goiás. Goiânia, GO, Brasil.

${ }^{3}$ Enfermeira. Doutora em Enfermagem. Docente de Enfermagem da Universidade Federal de Goiás. Goiânia, GO, Brasil.

Autor Correspondente:

Flaviane Cristina Rocha Cesar

Universidade Federal de Goiás

R. 227, s/n - 74605-080 - Goiânia, GO, Brasil

E-mail: flaviane_rocha01@hotmail.com
Recebido: $16 / 01 / 2018$

Finalizado: 03/10/2018 


\section{INTRODUÇÃO}

Estudantes de mestrado e doutorado frequentemente concebem o período da pós-graduação como altamente estressor ${ }^{(1)}$, estima-se que entre $38,9 \%$ e $90,6 \%$ percebam algum tipo de estressor durante a formação ${ }^{(2)}$. O estresse configura uma interação entre o indivíduo e o ambiente, interação essa considerada excedente aos seus recursos e uma ameaça ao seu bem-estar ${ }^{(3)}$. Causas de estresse relacionadas à formação dos pesquisadores são denominadas estressores acadêmicos, e a exposição deles a esses estressores relacionam-se a diversos resultados negativos ${ }^{(2)}$, tais como, prejuízos do desempenho dos acadêmicos ${ }^{(4)}$ e aumento do risco de adoecimento mental ${ }^{(5-6)}$.

As repercussões negativas à saúde resultantes da interação do indivíduo com os estressores dependem de sua capacidade adaptativa, e essa é influenciada por características biológicas, recursos psicossociais, padrão de coping aprendido, e do próprio estressor, no que se refere à natureza, número e persistência ${ }^{(7)}$.

Estudos sobre os estressores no ambiente acadêmico têm apontado seus possíveis prejuízos para além daqueles relacionados à saúde mental dos estudantes, podendo alcançar a qualidade da ciência produzida ${ }^{(8-9)}$, o que especialmente tem atingido novos pesquisadores ou pesquisadores em formação ${ }^{(8)}$.

A atividade dos estudantes de mestrado e doutorado constitui a principal fonte de avanço científico, quando se consideram o rigor e a inovação exigida do trabalho desenvolvido por eles ${ }^{(5)}$, e os altos níveis de estresse têm sido apontados como contraproducentes para a realização desse trabalho ${ }^{(9)}$. Nesse sentido, identificar os estressores é indispensável para a compreensão de sua relação com o processo saúde-doença dos estudantes de pós-graduação e fundamental para que gestores políticos e acadêmicos construam um ambiente saudável e produtivo.

Apesar da relevância da temática, das evidências apontarem o ambiente da pós-graduação como estressor $^{(1-2)}$ e da repercussão desse fator na produção da ciência, em revisão exploratória da literatura não foram encontrados estudos que descrevessem esses estressores de forma integrada ao processo de formação. Os estudos atuais limitam-se a identificar estressores de apenas um curso/programa ou país, tendo uma generalização limitada para serem usados na compreensão desse fenômeno.

Assim, a identificação dos estressores presentes no cotidiano dos pós-graduandos permite que os responsáveis pelos programas direcionem propostas que ajudem a reduzir o estresse acadêmico, colaborando para a redução de sintomas psicológicos negativos e para a construção de estratégias multimodais para o enfrentamento de estressores presentes durante a formação. Posto isso, o objetivo deste estudo foi identificar os estressores enfrentados por estudantes de mestrado e doutorado durante a sua formação.

\section{MÉTODO}

Revisão integrativa da literatura, operacionalizada por meio das seguintes etapas: determinação da questão de pesquisa; aplicação de critérios para a inclusão e exclusão de estudos; coleta de informações nos estudos selecionados; avaliação, interpretação, síntese e apresentação da revisão ${ }^{(10)}$. A questão norteadora foi: Quais são os estressores enfrentados por estudantes de mestrado e doutorado durante o seu processo de formação?

As buscas foram realizadas na Biblioteca Virtual em Saúde (BVS), Web of Science (interface com o portal CAPES), Psychology Information - PsycINFO (interface com o portal CAPES) e na US National Library of Medicine (PubMed). A seleção dos estudos foi realizada em outubro de 2017, por duas pesquisadoras em momentos diferenciados, a fim de constatar a homogeneidade da inclusão dos estudos.

A seleção dos estudos envolveu a combinação dos Descritores em Ciências da Saúde - DeCS (Educação de Pós-Graduação e Estresse Psicológico), termos da base PsycINFO (Stress e Postgraduate Students), termos Medical Subject Headings - MeSH (Education, Graduate e Stress, Psychological); e palavras-chave (student stressors, Phd student e doctoral student), conforme as especificidades de 
cada local de busca, a fim de garantir uma captação precisa e ampla de estudos, conforme disposto na Tabela 1.

Tabela 1 - Cruzamentos realizados na Biblioteca Virtual em Saúde e nas bases de dados Web of Science, PsyclNFO e PubMed. Goiânia, GO, Brasil, 2017

\begin{tabular}{ll} 
Local & Cruzamentos \\
\hline BVS & (tw:(Educação de Pós-Graduação)) AND (tw:(Estresse Psicológico)) \\
\hline PsycINFO & Any Field: \{Stress\} AND Any Field: \{Postgraduate Students\} \\
\hline PubMed & ("Education, Graduate"[Mesh] AND "Stress, Psychological"[Mesh]) \\
\cline { 2 - 2 } & ("Stress, Psychological"[Mesh]) AND ((phd student) OR doctoral student) \\
\hline Web of Science & Tópico: (Education, Graduate) AND Tópico: (Stress, Psychological) \\
\cline { 2 - 2 } & Tópico: ("student stressors") AND Tópico: ("phd student") OR Tópico: ("doctoral student")
\end{tabular}

Foram incluídos estudos observacionais e experimentais, de abordagem quantitativa e qualitativa, que apresentassem no título ou resumo o tema "estressores entre estudantes de mestrado e/ou doutorado", nos idiomas português, inglês e/ou espanhol, sem delimitação do ano de publicação, contemplaram-se artigos publicados até o mês da realização da busca on-line, em outubro de 2017.

Para compor a amostra, os artigos tinham de atender aos seguintes critérios de adequação ao tema: (I) contemplar apenas estudantes de pós-graduação (mestrado ou doutorado), ou, se outras amostras fossem consideradas, o estudo deveria fornecer informações sobre estudantes de pós-graduação separadamente e (II) conter informações sobre estressores em mestrandos e doutorandos. Foram excluídos artigos que fornecessem apenas medida global ou diagnóstico de estresse, estudos de revisão de literatura, artigos de opinião e relatos de caso.

A extração de dados dos artigos selecionados, para compor os resultados deste estudo, foi feita por meio de formulário previamente elaborado pelas autoras, com os tópicos: origem dos artigos (autor, ano, periódico e país), objetivo e público-alvo (objeto do trabalho), desenho metodológico e instrumento/estratégia de identificação dos estressores (metodologia) e os estressores identificados (estressores). O preenchimento foi realizado de forma independente pelas autoras, e, posteriomente, foram feitas 12 reuniões para chegar ao consenso sobre a construção do quadro-síntese e categorização dos resultados.

\section{RESULTADOS}

Inicialmente, na etapa de busca dos artigos, foram identificados 1.581 artigos com os cruzamentos dos termos de busca nas bases de dados utilizadas neste estudo. Depois da aplicação dos critérios de inclusão referentes ao idioma e tipo de documento restaram 1.347 manuscritos. Esses foram armazenados em um arquivo único no $\mathrm{EndNote}^{\circledR}$, e, por meio desse software, foram retiradas as duplas entradas dos artigos, resultantes da indexação de periódicos em mais de uma das bases de dados pesquisadas $(n=1.270)$.

Os artigos que contemplaram o tema da pesquisa no título ou resumo foram separados para serem lidos na íntegra $(n=34)$. Depois da leitura desses textos, foram verificados os critérios de adequação ao tema, restando 15 estudos, desses, três foram excluídos da amostra final por serem artigo de opinião $(n=2)$ e por apresentar apenas a prevalência do diagnóstico de estresse ( $n=1)$ (Figura 1). 


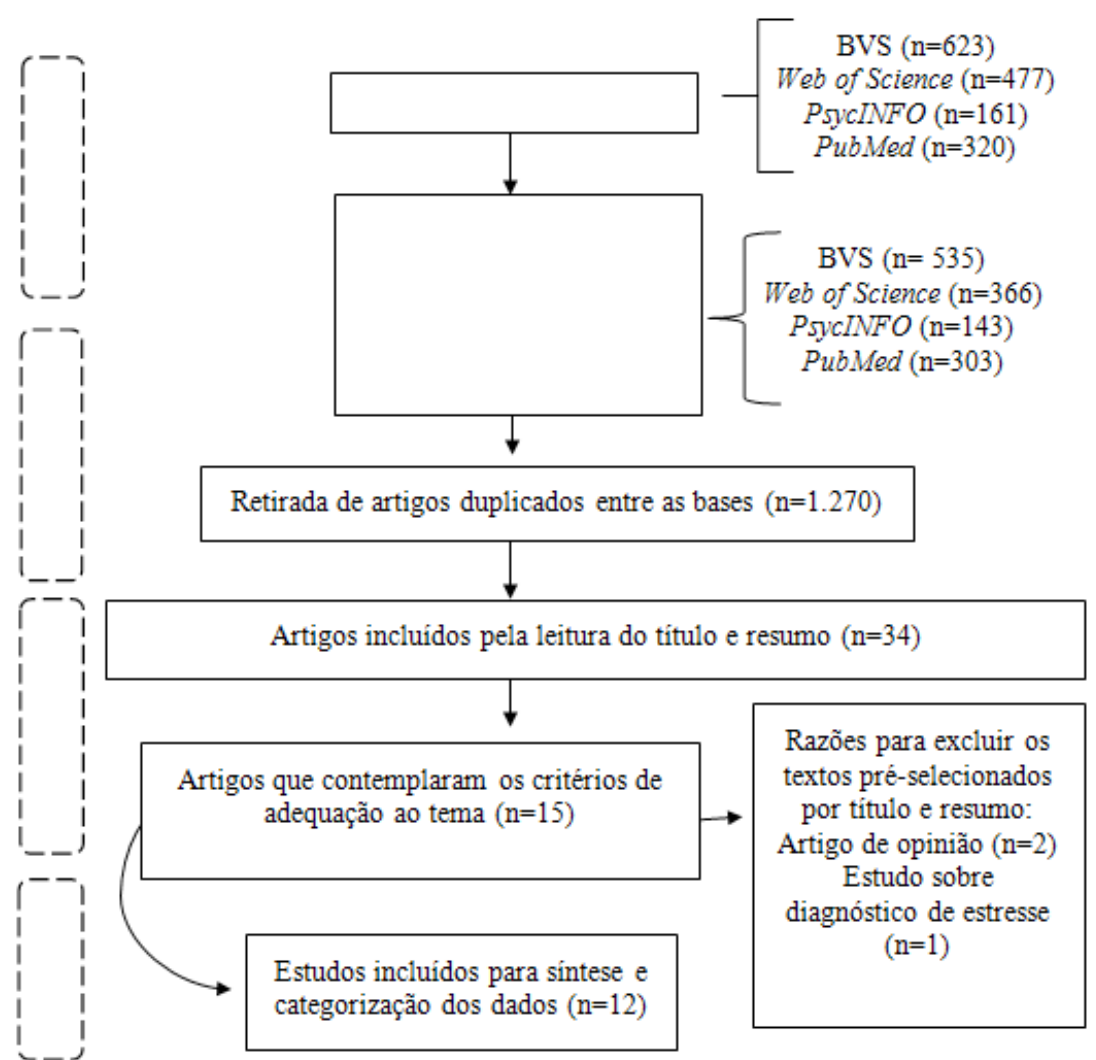

Figura 1 - Fluxograma referente à seleção dos estudos a serem incluídos na pesquisa, sobre os estressores enfrentados por estudantes de mestrado e doutorado durante o seu processo de formação, com base nos critérios preestabelecidos. Goiânia, GO, Brasil, 2017

Os resultados compuseram 12 artigos, com a data de publicação variando entre 1994 e 2016. Oito estudos $(66,7 \%)$ desenvolveram método quantitativo transversal ${ }^{(11-18)}$, e quatro $(33,3 \%)$, abordagem qualitativa ${ }^{(19-22)}$. A maioria dos estudos, seis (50,0\%), investigou os estressores por meio de questionários previamente validados ${ }^{(11,14-18)}$, seguida de estudos com questionários elaborados pelos autores ${ }^{(12-13,22)}$, roteiro de entrevista ${ }^{(19,21)}$ e áudio-diário ${ }^{(20)}$. Os estudantes de doutorado em psicologia foram os mais pesquisados, somando cinco publicações $(41,7 \%)^{(12,14,16-18)}$, e metade dos estudos foi realizado com discentes dos Estados Unidos ${ }^{(12,14-17,22)}$ (Quadro 1).

Quadro 1 - Características dos estudos que analisaram estressores entre estudantes de mestrado e doutorado. Goiânia, GO, Brasil, 2017 (continua)

\begin{tabular}{|c|c|c|c|}
\hline $\begin{array}{c}\text { Autor/periódico/ } \\
\text { país }\end{array}$ & Objeto do trabalho & Metodologia & Estressores \\
\hline $\begin{array}{c}\text { Brown K, } \\
\text { Anderson-Johnson } \\
\mathrm{P}, \\
\text { McPherson } \mathrm{A}^{(11)} \\
\text { Nurse Education in } \\
\text { Practice } \\
\text { Jamaica }\end{array}$ & $\begin{array}{l}\text { Níveis e estressores } \\
\text { acadêmicos e sua } \\
\text { relação com variáveis } \\
\text { sociodemográficas, } \\
\text { ano de curso e faixa } \\
\text { de especialidade com } \\
\text { estudantes do primeiro e } \\
\text { segundo ano de mestrado } \\
\text { em enfermagem. }\end{array}$ & $\begin{array}{l}\text { Estudo transversal, } \\
\text { que utilizou o } \\
\text { Questionário de } \\
\text { autorrelato de } \\
\text { estressores com } 45 \\
\text { itens. }\end{array}$ & $\begin{array}{l}\text { - Preparação para atividades } \\
\text { avaliativas das disciplinas } \\
\text { · Perspectiva do exame final da } \\
\text { pós-graduação } \\
\text { - Sobrecarga relacionada às } \\
\text { atividades da pós-graduação } \\
\text { · A exigência de escrever nos } \\
\text { padrões da pós-graduação } \\
\text {. Custos do curso }\end{array}$ \\
\hline
\end{tabular}




\begin{tabular}{|c|c|c|c|}
\hline $\begin{array}{l}\text { Devonport T, } \\
\text { Lane } \mathrm{A}^{(20)} \\
\text { Journal of Hospitality, } \\
\text { Leisure, Sport \& } \\
\text { Tourism Education } \\
\text { Inglaterra }\end{array}$ & $\begin{array}{l}\text { Identificação dos } \\
\text { estressores e estratégias } \\
\text { de enfrentamento entre } \\
\text { dois doutorandos em } \\
\text { ciências sociais no último } \\
\text { ano do doutorado e suas } \\
\text { respectivas parceiras. }\end{array}$ & $\begin{array}{l}\text { Pesquisa qualitativa } \\
\text { por meio de estudo } \\
\text { de caso com uso de } \\
\text { áudio-diário. }\end{array}$ & $\begin{array}{l}\text { - Dificuldade em priorizar os } \\
\text { compromissos do doutorado } \\
\text { - Incerteza sobre o futuro } \\
\text { profissional pós-academia } \\
\text { - Diminuição da renda familiar } \\
\text { para priorizar as atividades do } \\
\text { doutorado }\end{array}$ \\
\hline $\begin{array}{c}\text { Faro } \mathrm{A}^{(13)} \\
\text { Psicologia: Reflexão } \\
\text { e Crítica } \\
\text { Brasil }\end{array}$ & $\begin{array}{l}\text { As relações entre } \\
\text { estressores, estresse, } \\
\text { enfrentamento e bem- } \\
\text { estar subjetivo em pós- } \\
\text { graduandos no Brasil. }\end{array}$ & $\begin{array}{l}\text { Estudo transversal } \\
\text { (survey) por meio de } \\
\text { um Questionário } \\
\text { elaborado pelo } \\
\text { autor com } 15 \\
\text { preocupações } \\
\text { em relação à pós- } \\
\text { graduação. }\end{array}$ & $\begin{array}{l}\text { Compatibilizar os desafios } \\
\text { impostos pela formação e vida } \\
\text { pessoal } \\
\text { - Recursos financeiros insuficientes } \\
\text { relacionados ao tempo dedicado } \\
\text { aos estudos } \\
\text { · Atendimento às atividades } \\
\text { da pós-graduação no prazo } \\
\text { estabelecido } \\
\text { - Relacionamento aluno- } \\
\text { orientador quanto ao número e } \\
\text { aproveitamento dos encontros } \\
\text { - Sobrecarga relacionada à } \\
\text { produtividade, cobranças } \\
\text { individuais e do orientador } \\
\text { - Expectativa de desempenho nas } \\
\text { atividades do curso }\end{array}$ \\
\hline $\begin{array}{c}\text { Divaris K, } \\
\text { Polychronopoulou } \\
\text { A, Taoufik K, } \\
\text { Katsaros C, } \\
\text { Eliades T(18) } \\
\text { European journal of } \\
\text { dental education: } \\
\text { official journal of } \\
\text { the Association for } \\
\text { Dental Education in } \\
\text { Europe } \\
\text { Grécia }\end{array}$ & $\begin{array}{l}\text { Níveis de estresse e } \\
\text { Burnout percebido e } \\
\text { impacto do estresse } \\
\text { percebido em prática } \\
\text { odontológica entre } \\
\text { estudantes/ residentes } \\
\text { de pós-graduação nos } \\
\text { primeiros } 2 \text { anos de um } \\
\text { programa de Residência } \\
\text { que tem nível de ensino } \\
\text { de Mestrado e Doutorado. }\end{array}$ & $\begin{array}{l}\text { Estudo transversal, } \\
\text { realizado por meio } \\
\text { Graduate Dental } \\
\text { Environment Stress } \\
\text { questionnaire (GDES). }\end{array}$ & $\begin{array}{l}\text { - Falta de tempo para atividade de } \\
\text { lazer } \\
\text { · Questões financeiras } \\
\text { - Insegurança quanto ao futuro } \\
\text { profissional } \\
\text { - Negligência com a vida pessoal }\end{array}$ \\
\hline $\begin{array}{l}\text { El-Ghoroury } \\
\text { N, Galper D, } \\
\text { Sawaqdeh A, } \\
\text { Bufka L }{ }^{(12)} \\
\text { Training and } \\
\text { Education in } \\
\text { Professional } \\
\text { Psychology } \\
\text { Estados Unidos da } \\
\text { América, Distrito de } \\
\text { Colúmbia } \\
\text { e Porto Rico }\end{array}$ & $\begin{array}{l}\text { Estressores, estratégias de } \\
\text { enfrentamento e barreiras } \\
\text { ao uso de atividades de } \\
\text { bem-estar no último ano } \\
\text { do mestrado e do } 2^{\circ} \text { ao } \\
5^{\circ} \text { ano do doutorado em } \\
\text { psicologia. }\end{array}$ & $\begin{array}{l}\text { Estudo transversal } \\
\text { que utilizou dois } \\
\text { questionários } \\
\text { construídos pelos } \\
\text { autores: } \\
\text { 1) } 15 \text { preocupações } \\
\text { em relação à pós- } \\
\text { graduação; } \\
\text { 2) Questionário } \\
\text { estruturado com } 22 \\
\text { itens abrangendo } \\
\text { eventos de vida } \\
\text { maiores e menores, } \\
\text { principais estressores } \\
\text { acadêmicos e } \\
\text { interpessoais de } \\
\text { estudantes de } \\
\text { pós-graduação em } \\
\text { psicologia. }\end{array}$ & $\begin{array}{l}\text { - Pressão por responsabilidades } \\
\text { acadêmicas } \\
\text { · Custos do curso } \\
\text { · Ansiedade } \\
\text { - Desequilíbrio entre trabalho } \\
\text { clínico e vida acadêmica }\end{array}$ \\
\hline
\end{tabular}




\begin{tabular}{|c|c|c|c|}
\hline $\begin{array}{c}\text { Jungbluth C, } \\
\text { MacFarlane I, Veach } \\
\text { P, } \\
\text { LeRoy B }{ }^{(15)} \\
\text { Journal of Genetic } \\
\text { Counseling } \\
\text { Estados Unidos da } \\
\text { América e Canadá }\end{array}$ & $\begin{array}{l}\text { Experiência de ansiedade, } \\
\text { tipos de estressores e } \\
\text { estratégias recomendadas } \\
\text { para o gerenciamento dos } \\
\text { estressores em estudantes } \\
\text { de mestrado em } \\
\text { aconselhamento genético. }\end{array}$ & $\begin{array}{l}\text { Estudo transversal } \\
\text { que utilizou o } \\
\text { Questionário } \\
\text { adaptado sobre bem- } \\
\text { estar em ambientes } \\
\text { acadêmicos. }\end{array}$ & $\begin{array}{l}\text { - Falta de tempo para interagir com } \\
\text { a família e os amigos } \\
\text { - Relação com o orientador/ } \\
\text { supervisor que envolve situações } \\
\text { de humilhação e abuso } \\
\text { - Conflito e competição com } \\
\text { colegas da pós-graduação } \\
\text { - Carga de emoções relacionadas } \\
\text { ao contato com pacientes } \\
\text { - Falta de confiança, conflito moral } \\
\text { e autocobrança } \\
\text { - Dificuldade em conciliar a } \\
\text { elaboração do projeto da tese } \\
\text { com outras atividades acadêmicas, } \\
\text { profissionais e pessoais } \\
\text { - Desequilíbrio entre a quantidade } \\
\text { de recursos financeiros disponíveis } \\
\text { e a quantidade de dívidas } \\
\text { - Incerteza sobre um possível } \\
\text { emprego após finalizar a pesquisa } \\
\text { - Falta de tempo para interagir } \\
\text { com pessoas e ambientes não } \\
\text { acadêmicos }\end{array}$ \\
\hline $\begin{array}{l}\text { Bujdoso Y, Cohn } \\
\qquad \mathrm{A}^{(19)} \\
\text { Revista de Saúde } \\
\text { Pública } \\
\text { Brasil, SP }\end{array}$ & $\begin{array}{l}\text { A relação entre os } \\
\text { principais indícios } \\
\text { de estresse, coping } \\
\text { e estressores em } \\
\text { mestrandos enfermeiros e } \\
\text { o processo de elaboração } \\
\text { da dissertação com sua } \\
\text { inserção profissional. }\end{array}$ & $\begin{array}{l}\text { Pesquisa qualitativa, } \\
\text { com roteiro } \\
\text { de entrevistas } \\
\text { semiestruturadas. }\end{array}$ & $\begin{array}{l}\text { - Dificuldade de conciliar } \\
\text { mestrado, trabalho assistencial, lar } \\
\text { e vida pessoal } \\
\text { - Dificuldade de lidar com prazo } \\
\text { de conclusão }\end{array}$ \\
\hline $\begin{array}{l}\text { Pfeifer T, Kranz } \mathrm{P}, \\
\text { ScogginA }{ }^{(22)} \\
\text { Occupational } \\
\text { Therapy International } \\
\text { Estados Unidos da } \\
\text { América, Texas }\end{array}$ & $\begin{array}{l}\text { O estresse percebido em } \\
\text { estudantes do primeiro e } \\
\text { segundo ano do mestrado } \\
\text { em terapia ocupacional } \\
\text { (TO) de uma universidade } \\
\text { no Texas. }\end{array}$ & $\begin{array}{l}\text { Método misto, com } \\
\text { etapas qualitativas } \\
\text { e estatísticas } \\
\text { descritivas } \\
\text { realizadas por meio } \\
\text { de instrumento } \\
\text { elaborado pelos } \\
\text { próprios autores, } \\
\text { baseados na } \\
\text { literatura. }\end{array}$ & $\begin{array}{l}\text { Dificuldade de gerenciamento } \\
\text { de tempo para equilibrar estudo, } \\
\text { família e trabalho } \\
\text { - Dificuldade de gerir a carga de } \\
\text { trabalho acadêmica } \\
\text { - Falta de clareza no curso, nas } \\
\text { expectativas e no programa }\end{array}$ \\
\hline $\begin{array}{c}\text { McKinzie C, } \\
\text { Altamura V, } \\
\text { Burgoon E, Bishop } \\
\text { C(16) } \\
\text { Psychological reports } \\
\text { Estados Unidos } \\
\text { da América, Nova } \\
\text { Iorque }\end{array}$ & $\begin{array}{l}\text { Avaliou o papel de } \\
\text { preditor do estresse } \\
\text { das variáveis hábitos } \\
\text { diários, autoestima e } \\
\text { humor, por meio da } \\
\text { análise de correlação } \\
\text { entre estudantes de pós- } \\
\text { graduação em psicologia. }\end{array}$ & $\begin{array}{l}\text { Estudo transversal } \\
\text { analítico que utilizou } \\
\text { a Student Stress Scale } \\
\text { (Insel \& Roth, 1991). }\end{array}$ & $\begin{array}{l}\text { - Poucas horas de sono } \\
\text { - Sentimentos negativos }\end{array}$ \\
\hline $\begin{array}{c}\text { Meis L,Velloso } \\
\text { A,Lannes D,Carmo } \\
\text { M,Meis C (21) } \\
\text { Brazilian Journal } \\
\text { of Medical and } \\
\text { Biological Research } \\
\text { Brasil, Rio de } \\
\text { Janeiro }\end{array}$ & $\begin{array}{l}\text { Entrevistas com } \\
\text { estudantes de pós- } \\
\text { graduação e professores } \\
\text { de um departamento de } \\
\text { bioquímica para analisar a } \\
\text { ciência no Brasil. }\end{array}$ & $\begin{array}{l}\text { Estudo qualitativo, } \\
\text { entrevistas abertas } \\
\text { e } 124 \text { entrevistas } \\
\text { semiestruturadas } \\
\text { por meio de um } \\
\text { instrumento } \\
\text { elaborado pelos } \\
\text { próprios autores. }\end{array}$ & $\begin{array}{l}\text { - Sobrecarga de atividades } \\
\text { acadêmicas } \\
\text { - Competição entre os pares } \\
\text { acadêmicos } \\
\text { - Dificuldades de financiamento } \\
\text { para a pesquisa } \\
\text { - Incerteza sobre a carreira } \\
\text { acadêmica } \\
\text { - Momentos de avaliações } \\
\text { acadêmicas }\end{array}$ \\
\hline
\end{tabular}




\begin{tabular}{|c|c|c|c|}
\hline $\begin{array}{l}\text { Nelson N, } \\
\text { Dell'Oliver C, Koch } \\
\text { C, Buckler } \mathrm{R}^{(17)} \\
\text { Psychological reports } \\
\text { Estados Unidos da } \\
\text { América, Chicago }\end{array}$ & $\begin{array}{l}\text { O estudo focou a } \\
\text { verificação da interação } \\
\text { entre estresse, sofrimento, } \\
\text { saúde, apoio social e } \\
\text { utilização de estratégias } \\
\text { de enfrentamento e } \\
\text { sucesso acadêmico em } \\
\text { estudantes de doutorado } \\
\text { em psicologia clínica. }\end{array}$ & $\begin{array}{l}\text { Estudo transversal } \\
\text { (survey) que utilizou } \\
\text { o Demographic/Stress } \\
\text { Questionnaire para } \\
\text { avaliar os estressores } \\
\text { da pós-graduação. }\end{array}$ & $\begin{array}{l}\text { - Atividades do curso e o } \\
\text { desenvolvimento da dissertação } \\
\text { - Situação financeira pessoal } \\
\text { - Expectativas relacionadas à } \\
\text { prática assistencial } \\
\text { - Aborrecimentos diários } \\
\text { - Gerenciamento de tempo } \\
\text { - Contato com o paciente }\end{array}$ \\
\hline $\begin{array}{l}\text { Hudson S, O'Regan } \\
\qquad J^{(14)} \\
\text { Journal of clinical } \\
\text { psychology } \\
\text { Estados Unidos da } \\
\text { América, Minnesota }\end{array}$ & $\begin{array}{l}\text { O estudo examinou se } \\
\text { gênero do aluno; ano no } \\
\text { programa; nível de renda; } \\
\text { status do relacionamento; } \\
\text { idade; número de filhos; } \\
\text { número de horas de } \\
\text { emprego são fatores } \\
\text { relacionados ao estresse } \\
\text { em pós-graduandos de } \\
\text { psicologia. }\end{array}$ & $\begin{array}{l}\text { Estudo transversal } \\
\text { que utilizou } \\
\text { Psychology Student } \\
\text { Stress Questionnaire } \\
\text { (Cahir \& Morris, } \\
\text { 1991). }\end{array}$ & $\begin{array}{l}\text { Neste estudo, dois conjuntos de } \\
\text { características sociodemográficas } \\
\text { foram arroladas como estressoras: } \\
\text { · Ter filhos e não ter um } \\
\text { relacionamento estável durante a } \\
\text { pós-graduação } \\
\text { · Mulheres que trabalham em } \\
\text { tempo integral e que não têm } \\
\text { relacionamento estável }\end{array}$ \\
\hline
\end{tabular}

Os estressores elencados no Quadro 1 foram posteriormente distribuídos em três categorias, sendo essas: características individuais como estressoras na formação de mestres e doutores; atividades acadêmicas como estressoras na pós-graduação; e relações interpessoais como estressoras durante a pós-graduação.

No contexto das características individuais, em 11 artigos (91,6\%), foram evidenciadas características particulares dos estudantes que têm papel de estressor no contexto da pós-graduação. Esses estressores corresponderam a dificuldades financeiras ${ }^{(11-13,17-18,21)}$, insegurança sobre o futuro profissional ${ }^{(15,18,20-21)}$, gênero feminino ${ }^{(14)}$, poucas horas de sono ${ }^{(15-16)}$, sentimentos negativos ${ }^{(16)}$ e ansiedade $^{(12)}$. Destaca-se que em mais da metade dos estudos avaliados nesta revisão, recursos financeiros insuficientes constituíram estressores individuais relevantes ${ }^{(11-13,17-19)}$, com frequência variando entre $26,2 \%$ e $63,9 \%$ nas amostras estudadas $^{(11-13,23)}$. Para os demais estressores dessa categoria, os estudos não estabeleceram frequência, mas identificaram forte associação com o diagnóstico de estresse ${ }^{(16,18)}$ e traço de ansiedade ${ }^{(15)}$.

No âmbito das atividades acadêmicas, quatro (33,3\%) artigos evidenciaram que os momentos nos quais se intensifica o uso das habilidades cognitivas dos estudantes culminam em estressores significativos $^{(11,13,15,17)}$. Exemplos desses momentos são a preparação para atividades avaliativas das disciplinas, perspectiva do exame final da pós-graduação, exigência de escrever nos padrões da pós-graduação(11), expectativa de desempenho nas atividades do curso ${ }^{(13)}$, demandas do curso e desenvolvimento da dissertação(17) e trabalho acadêmico(15). Os estressores dessa categoria atingiram entre $68,1 \%$ e $73,7 \%$ dos estudantes avaliados ${ }^{(11-12)}$.

Na categoria atividades acadêmicas, estressores ligados à alta quantidade ou número de tarefas, superior à capacidade dos estudantes, foram elencados em sete $(58,3 \%)$ artigos. Foram incluídas a dificuldade de gerir a carga de trabalho acadêmica ${ }^{(22)}$, de atender às atividades da pós-graduação no prazo estabelecido(13,19), em priorizar os compromissos do doutorado(20), sobrecarga relacionada às atividades acadêmicas ${ }^{(13,21)}$ e pressão por responsabilidades acadêmicas, relatada por $68,1 \%$ dos estudantes ${ }^{(12) .}$

A categoria "estressores das relações interpessoais" foi construída por quatro (33,3\%) artigos e compreendeu situações de conflito no ambiente acadêmico, caracterizadas pela dificuldade de relacionamento com colegas de curso e com o orientador ${ }^{(15,21,24)}$, atingindo de $12,3 \%$ a $23,7 \%{ }^{(24)}$ dos estudantes e, no ambiente extra-acadêmico, dificuldade de conciliar a pós-graduação com o tempo para familiares e amigos ${ }^{(15,24)}$, referida por $65,2 \%$ dos estudantes avaliados ${ }^{(13)}$. 


\section{DISCUSSÃO}

Busca sistemática nas principais bases de dados da área da saúde permitiu identificar número de tarefas acadêmicas maior do que a capacidade dos estudantes, dificuldades financeiras, conflito da pós-graduação com a família e trabalho e dificuldades na relação com o orientador como os principais estressores que incidem sobre estudantes de mestrado e doutorado durante a sua formação. Nesta revisão, indicaram-se categorias de ação para que as políticas de saúde, os programas de pós-graduação e os próprios estudantes possam implementar medidas que diminuam ou atenuem a presença dos estressores no meio acadêmico.

O número de tarefas maior do que a capacidade dos estudantes de mestrado e doutorado foi o estressor principal da categoria "Atividades acadêmicas como estressoras na pós-graduação". A falha na gestão das tarefas é apontada em diferentes estudos como dificuldades na articulação das atividades da pós-graduação com necessidades pessoais, familiar e profissionais ${ }^{(12-13,15,17-20,22)}$.

Embora se reconheça que a capacidade de executar tarefas acadêmicas possa estar relacionada a fatores individuais, como falta/falha de planejamento, é necessário considerar também o papel de aspectos externos aos indivíduos, como os intensos compromissos acadêmicos ${ }^{(21)}$. Dessa forma, a gestão das atividades acadêmicas pode ser considerada reflexo da tendência de as instituições privilegiarem a produtividade ${ }^{(9)}$, exigindo de mestrandos e doutorandos maior demanda de tempo para o envolvimento nas tarefas acadêmicas ${ }^{(11-13,16)}$.

Além de ser um estressor relevante para os estudantes, as práticas avaliativas que privilegiam a quantidade em vez da qualidade e o impacto social das produções científicas podem trazer efeito negativo sobre a imaginação e inovação em pesquisas ${ }^{(8)}$. Tais práticas favorecem também a má prática em publicação, como plágio e publicação duvidosa de resultados ${ }^{(9)}$.

É preciso uma mudança coletiva da ótica de trabalho do pós-graduando, deixando-se de priorizar apenas o caráter quantitativo da produção científica, valorizando também os aspectos qualitativos na formação dos novos cientistas ${ }^{(9)}$. Além disso, é necessário que os programas discutam e viabilizem o planejamento da vida acadêmica durante o curso, oportunamente ainda no primeiro ano acadêmico, fortalecendo as redes de apoio, com disciplinas integradoras e grupos de pesquisa, oferecendo workshops sobre planejamento da vida acadêmica ${ }^{(15,17)}$ e serviços de suporte, como atendimento especializado $^{(12,15,18)}$.

De um ponto de vista complementar, o conflito pós-graduação, família e trabalho e a relação com o orientador foram destaques entre os estressores da categoria "Relações interpessoais como estressoras durante a pós-graduação". Os estudantes frequentemente relatam que têm dificuldade em conciliar a pós-graduação com a família, com os amigos e com a vida pessoal(13,15,19,22), inclusive de cuidar de si mesmo $^{(18)}$. Além disso, a competição incentivada no ambiente acadêmico é marcada por conflito ${ }^{(15,21)}$ e vai contra a colaboração conjunta e o trabalho em equipe, que são apontados como estratégias de coping aos estressores acadêmicos ${ }^{(12)}$.

Nesse cenário, ascende também o papel da relação aluno-orientador, que pode atuar potencializando ${ }^{(13)}$ ou minimizando o estresse ${ }^{(1)}$. O número de contato ${ }^{(23)}$ e o apoio para o aprendizado fornecido pelo orientador ${ }^{(24-25)}$ estão envolvidos com o caráter positivo ou negativo dessa relação. Além disso, o tipo de liderança exercido pelo orientador ${ }^{(5)}$ e situações de abuso e/ou humilhações são consideradas pelos estudantes como estressoras ${ }^{(15)}$.

A importância de compreender a rede de relacionamentos, dentro da qual os indivíduos existem, emerge da constatação de que o "eu" é constituído fundamentalmente por um processo relacional ${ }^{(26)}$. Além disso, o papel das relações interpessoais tem influência na habilidade dos seres humanos em lidar com os estressores, podendo atuar como suporte social ${ }^{(27)}$, que ajuda na construção de respostas positivas a estressores, ou na promoção de estressores, como conflitos e competição ${ }^{(15,21)}$.

Os programas de pós-graduação podem implementar e fomentar algumas estratégias que fortaleçam as relações interpessoais dos estudantes, uma vez que essas auxiliam nas estratégias de coping do estresse ${ }^{(28)}$. Entre elas, destacam-se a promoção de condições de apoio mútuo entre alunos com o fortalecimento de grupos de pesquisas, a troca de experiências, a formação de vínculos entre 
os alunos da pós-graduação em níveis de formação diferentes e o desenvolvimento de liderança nos docentes da instituição.

Outro ponto de alerta destacado nesta revisão foi o recurso financeiro, na categoria "Características individuais como estressoras na formação de mestres e doutores". A renda constituiu-se em um estressor importante para pós-graduandos do Brasil ${ }^{(23-24)}$, da Inglaterra ${ }^{(20)}$, dos Estados Unidos ${ }^{(12,17)}$, da Grécia $^{(18)}$, da Jamaica ${ }^{(11)}$ e do Irã ${ }^{(1)}$.

No estudo brasileiro, o estressor renda foi mais significativo, quando comparado aos resultados dos demais países, podendo estar relacionado ao número e valor insuficiente da bolsas ofertadas no Brasil para os estudantes ${ }^{(24)}$. A característica da distribuição das bolsas e do financiamento da pesquisa brasileira pode explicar também o porquê de o vínculo empregatício, uma fonte direta de renda, aparecer como atenuador do estresse entre os pós-graduandos brasileiros ${ }^{(24)}$.

Outra faceta do vínculo empregatício é o estresse decorrente do conflito relacionado à articulação do emprego com as atividades acadêmicas ${ }^{(12,19,22)}$, mas esse efeito não é uma regra. Para os estudantes de mestrado em enfermagem, por exemplo, o trabalho assistencial foi mais estressante que a pósgraduação, e o ambiente acadêmico tornou-se mecanismo de fuga e busca de suporte para lidar com o estresse relacionado à prática assistencial ${ }^{(19)}$.

Em resumo, as categorias de estressores construídas neste estudo elencam os principais aspectos que podem ter efeito negativo na vida acadêmica e saúde dos estudantes de mestrado e doutorado. Revisão de literatura recente sustenta categorias semelhantes às deste estudo, apontando fatores pessoais, fatores relacionais e fatores institucionais da adaptação à pós-graduação ${ }^{(29)}$. Esse dado nos permite inferir que a relação do estudante com a pós-graduação é multicausal, influenciada pelo menos por três fatores: individuais, acadêmicos e de relações interpessoais.

Nesse contexto, apesar das limitações relacionadas à estratégia de busca, que pode não ter identificado todos os estudos publicados, na revisão atual conseguiu-se identificar os estressores relevantes e acrescentar um escopo de conhecimento sobre a experiência acadêmica a partir de evidências da literatura.

\section{CONSIDERAÇÕES FINAIS}

Os estudos identificados nesta revisão demonstram que os estudantes de mestrado e doutorado são expostos a estressores relacionados às características individuais, às atividades acadêmicas e às relações interpessoais durante a sua formação.

A alta quantidade de atividades acadêmicas constituiu o estressor acadêmico mais relevante nos estudos analisados, sendo correlacionada com os demais estressores. Os recursos financeiros foram elencados como relevante estressor individual e obtiveram maior impacto no Brasil. O conflito pósgraduação, família e trabalho e a relação com o orientador foram destaque entre os estressores das relações interpessoais.

O estresse produtivo e desafiador que conduz o estudante a efetivar as demandas acadêmicas é positivo e inerente ao processo de formação de mestres e doutores. É necessário, porém, estabelecer estratégias que favoreçam a adaptação desses estudantes, de modo a minimizar prejuízos à saúde mental e na vida extra-acadêmica. O caminho para a construção de um ambiente mais saudável passa pela busca de estudos factíveis, realizáveis, e que a qualidade tenha valor superior à quantidade. Além disso, ascende a necessidade de discutir o quanto os programas de pós-graduação podem atuar na construção desse ambiente.

O grande desafio para o pós-graduando é conciliar o estresse vivido nesse momento da vida, as atividades que a pós-graduação exige e as demandas sociais. Os programas de pós-graduação precisam equilibrar os processos avaliativos existentes com a produção científica de qualidade, para que não resulte no adoecimento dos envolvidos nesse processo.

O sucesso das pesquisas e descobertas científicas, buscado pelos países ao redor do mundo, talvez, não esteja nas métricas e investimentos astronômicos, mas na simples garantia do livre pensar 
para os atores envolvidos no processo de construção da ciência. A pós-graduação precisa ser vista como trabalho intelectual que impulsiona o bem social e, com isso, entendermos que o maior valor durante esse processo se relaciona ao capital humano. Sem essa compreensão, o risco de prejudicar os avanços científicos e os benefícios que a ciência pode trazer para a sociedade continuará presente.

\section{REFERÊNCIAS}

1. Bazrafkan L, Shokrpour N, Yousefi A, Yamani N. Management of Stress and Anxiety Among PhD Students During Thesis Writing: A Qualitative Study. Health Care Manag. [Internet]. 2016 [acesso em 2017 dez 06]; 35(3). Disponível em: https://dx.doi.org/10.1097/hcm.0000000000000120.

2. Beall JW, DeHart RM, Riggs RM, Hensley J. Perceived Stress, Stressors, and Coping Mechanisms among Doctor of Pharmacy Students. Pharmacy. [Internet]. 2015 [acesso em 2017 dez 07]; 3(4). Disponível em: http://doi. org/10.3390/pharmacy3040344.

3. Lazarus RS, Folkman S. The Stress Concept in the Life Sciences. In: Lazarus RS, organizador. Stress, appraisal, and coping. New York: Springer Pub. Co.; 1984. p. 1-21.

4. Hubbard KK, Blyler D. Improving Academic Performance and Working Memory in Health Science Graduate Students Using Progressive Muscle Relaxation Training. Am J Occup Ther. [Internet]. 2016 [acesso em 2017 dez 08]; 70(6). Disponível em: http://ajot.aota.org/article.aspx?articleid=2569270.

5. Levecque K, Anseel F, De Beuckelaer A, Van der Heyden J, Gisle L. Work organization and mental health problems in PhD students. Res. Policy. [Internet]. 2017 [acesso em 2017 dez 06]; 46(4). Disponível em: http://doi. org/10.1016/j.respol.2017.02.008.

6. O'Reilly E, McNeill KG, Mavor KI, Anderson K. Looking Beyond Personal Stressors: An Examination of How Academic Stressors Contribute to Depression in Australian Graduate Medical Students. Teach. Learn. Med. [Internet]. 2014 [acesso em 2017 dez 06] ; 26(1). Disponível em: http://dx.doi.org/10.1080/10401334.2013.857330.

7. Schneiderman N, Ironson G, Siegel SD. Stress and Health: Psychological, Behavioral, and Biological Determinants. Annu. Ver. Clin. Psychol. [Internet]. 2004 [acesso em 2017 jun 06]; 1(1). Disponível em: http://dx.doi.org/10.1146/ annurev.clinpsy.1.102803.144141.

8. Fochler M, Felt U, Muller R. Unsustainable Growth, Hyper-Competition, and Worth in Life Science Research: Narrowing Evaluative Repertoires in Doctoral and Postdoctoral Scientists' Work and Lives. Minerva [Internet]. 2016 [acesso em 2017 dez 06]; 54(2). Disponível em: https://doi.org/10.1007/s11024-016-9292-y.

9. Tijdink JK, Schipper K, Bouter LM, Pont PM, de Jonge J, Smulders YM. How do scientists perceive the current publication culture? A qualitative focus group interview study among Dutch biomedical researchers. BMJ Open. [Internet]. 2016 [acesso em 2017 dez 06]; 6(2). Disponível em: https://doi.org/10.1136/bmjopen-2015-008681.

10. Whittemore R, Knafl K. The integrative review: updated methodology. J. Adv. Nurs. [Internet]. 2005 [acesso em 2017 jun 06]; 52(5) Disponível em: http://dx.doi.org/10.1111/j.1365-2648.2005.03621.x.

11. Brown K, Anderson-Johnson P, McPherson AN. Academic-related stress among graduate students in nursing in a Jamaican school of nursing. Nurse Educ. Pract. [Internet]. 2016 [acesso em 2017 dez 06]; 20(1). Disponível em: https://doi.org/10.1016/j.nepr.2016.08.004.

12. El-Ghoroury NH, Galper DI, Sawaqdeh A, Bufka LF. Stress, Coping, and Barriers to Wellness Among Psychology Graduate Students. Train Educ Prof Psychol [Internet]. 2012 [acesso em 2017 dez 06]; 6(2). Disponível em: http:// dx.doi.org/10.1037/a0028768.

13. Faro A. An Explanatory Model for Subjective Well-Being: A Study with Masters and PhD Students in Brazil. Psicol. Reflex. Crit. [Internet]. 2013 [acesso em 2017 dez 06]; 26(4). Disponível em: http://dx.doi.org/10.1590/S010279722013000400005.

14. Hudson SA, O'Regan J. Stress and the graduate psychology student. J. Clin. Psychol. [Internet]. 1994 [acesso em 2017 jun 06]; 50(6). Disponível em: https://doi.org/10.1002/1097-4679(199411)50:6<973::AIDJCLP2270500623>3.0.CO;2-Q. 
15. Jungbluth C, MacFarlane IM, Veach PM, LeRoy BS. Why is Everyone So Anxious?: An Exploration of Stress and Anxiety in Genetic Counseling Graduate Students. J Genet Couns [Internet]. 2011 [acesso em 2017 dez 06]; 20(3). Disponível em: https://doi.org/10.1007/s10897-010-9348-3.

16. McKinzie C, Altamura V, Burgoon E, Bishop C. Exploring the effect of stress on mood, self-esteem, and daily habits with psychology graduate students. Psychol. Rep. [Internet]. 2006 [acesso em 2017 dez 11]; 99(2) Disponível em: https://doi.org/10.2466/pr0.99.2.439-448.

17. Nelson NG, Dell'Oliver C, Koch C, Buckler R. Stress, coping, and success among graduate students in clinical psychology. Psychol Rep. [Internet]. 2001 [acesso em 2017 dez 06]; 88. Disponível em: https://doi.org/10.2466/ pr0.2001.88.3.759.

18. Divaris K, Polychronopoulou A, Taoufik K, Katsaros C, Eliades T. Stress and burnout in postgraduate dental education. Eur J Dent Educ [Internet]. 2012 [acesso em 2017 jun 06]; 16(1). Disponível em: https://doi.org/10.1111/ j.1600-0579.2011.00715.x.

19. Bujdoso YLV, Cohn AC. University as coping for dealing with care work of nursing Master's students. Rev. Saúde Públ. [Internet]. 2008 [acesso em 2017 dez 11]; 42(2). Disponível em: http://dx.doi.org/10.1590/S003489102008000200012.

20. Devonport TJ, Lane AM. In it together: Dyadic coping among doctoral students and partners. JoHLSTE. [Internet]. 2014 [acesso em 2017 dez 06]; 15(1). Disponível em: https://doi.org/10.1016/j.jhlste.2014.08.002.

21. Meis L, Velloso A, Lannes D, Carmo MS, Meis C. The growing competition in Brazilian science: rites of passage, stress and burnout. Braz J Med Biol Res. [Internet]. 2003 [acesso em 2017 jun 06]; 36(9). Disponível em: http:// www.ncbi.nlm.nih.gov/pubmed/12937778.

22. Pfeifer TA, Kranz PL, Scoggin AE. Perceived stress in occupational therapy students. Occupational Therapy International. [Internet]. 2008 [acesso em 2017 dez 06]; 15(4). Disponível em: https://doi.org/10.1002/oti.256.

23. Santos AF, Alves Júnior A. Estresse e estratégias de enfrentamento em mestrandos de ciências da saúde. Psicol. Reflex. Crit. [Internet]. 2007 [acesso em 2017 dez 11]; 20(1). Disponível em: http://dx.doi.org/10.1590/S010279722007000100014.

24. Faro A. Estresse e estressores na pós-graduação: estudo com mestrandos e doutorandos no Brasil. Psic.: Teor e Pesq. [Internet]. 2013 [acesso em 2017 dez 11]; 29(1). Disponível em: http://dx.doi.org/10.1590/S010237722013000100007.

25. Stubb J, Pyhalto K, Lonka K. Balancing between inspiration and exhaustion: PhD students' experienced sociopsychological well-being. Stud. Contin Educ. [Internet]. 2011 [acesso em 2017 dez 06]; 33(1). Disponível em: https://doi.org/10.1080/0158037X.2010.515572.

26. Roseneil S, Ketokivi K. Relational Persons and Relational Processes: Developing the Notion of Relationality for the Sociology of Personal Life. Sociology. [Internet]. 2016 [acesso em 2017 dez 07]; 50(1). Disponível em: https:// doi.org/10.1177/0038038514561295.

27. Baqutayan S. Stress and Social Support. Indian J Psychol Med. [Internet] 2011;33(1) [acesso em 09 dez 2017]. Disponível: http://dx.doi.org/10.4103/0253-7176.85392.

28. Canesqui AM, Barsaglini RA. Apoio social e saúde: pontos de vista das ciências sociais e humanas. Ciênc. saúde colet. [Internet]. 2012 [acesso em 2017 dez 02]; 17(1). Disponível em: http://dx.doi.org/10.1590/S141381232012000500002.

29. Santos AS, Perrone CM, Dias ACG. Adaptação à pós-graduação stricto sensu: uma revisão sistemática de literatura. Psico-USF. [Internet]. 2015 [acesso em 2017 dez 06]; 20(1). Disponível em: http://dx.doi.org/10.1590/141382712015200113. 